\title{
Five-Month-Old Infants Attribute Inferences Based on General Knowledge to Agents
}

\author{
Fransisca Ting ${ }^{\mathrm{a}, 1}$, Zijing He ${ }^{\mathrm{b}, 1}$, and Renée Baillargeon ${ }^{\mathrm{a}, 1}$
}

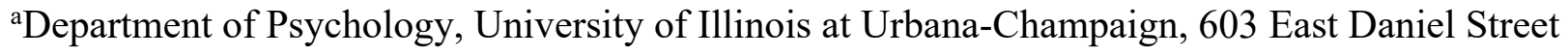
Champaign, IL 61820, USA

bepartment of Psychology, Sun Yat-sen University, 135 Xingangxi Road, Guangzhou, Guangdong 510275, China

${ }^{1}$ To whom correspondence may be addressed: fting@bu.edu, hezij2@mail.sysu.edu.cn, or rbaillar@illinois.edu.

Word count (maximum 10, 500): 8,525

Acknowledgments: This research was supported by a grant from the John Templeton Foundation to R. B. (60847) and by a grant from the National Natural Science Foundation of China to Z. H. (31200763). We thank the UIUC Infant Cognition Lab for their help with the data collection; graphic artist Steve Holland for producing the figures; and the families who participated in the research. 


\section{Highlights}

- Young infants track what information agents can obtain through perception.

- We examined if they also track what information agents can obtain through inference.

- 5-month-olds expected an agent to search for a wide toy hidden in her absence in a wide vs. a narrow box.

- This result shows that early psychological reasoning is not cognitively encapsulated.

- Infants can integrate other cognitive processes into their psychological reasoning. 


\begin{abstract}
To make sense of others' actions, we generally consider what information is available to them. This information may come from different sources, including perception and inference. Like adults, young infants track what information agents can obtain through perception: If an agent directly observes an event, for example, they expect her to have information about it. However, no investigation has yet examined whether young infants also track what information agents can obtain through inference, by bringing to bear relevant general knowledge. Building on the finding that by 4 months of age, most infants have acquired the physical rule that wide objects can fit into wide but not narrow containers, we asked whether 5-month-olds would expect an agent who was searching for a wide toy hidden in her absence to reach for a wide as opposed to a narrow box. Infants looked significantly longer when the agent selected the narrow box, suggesting that they expected her (a) to share the physical knowledge that wide objects can fit only into wide containers and (b) to infer that the wide toy must be hidden in the wide box. Three additional conditions supported this interpretation. Together, these results cast doubt on two-system accounts of early psychological reasoning, which claim that infants' early-developing system is too inflexible and encapsulated to integrate inputs from other cognitive processes, such as physical reasoning. Instead, the results support one-system accounts and provide new evidence that young infants' burgeoning psychological-reasoning system is qualitatively similar to that of older children and adults.
\end{abstract}

Keywords: infancy, theory of mind, knowledge, inference, two-system accounts, preference 


\section{Introduction}

To predict and interpret agents' actions, we generally need to consider what information is available to them. Agents may gather information from a number of different sources, including perception and inference. To illustrate, imagine the following scene: We are in a park enjoying the antics of a young girl and her large dog. At some point, the dog becomes hidden behind one of two bushes. If the girl saw the dog disappear behind the bush or heard it bark once there, we would expect her to know its location, through perception. If she did not watch the dog disappear or hear it bark but could see that one of the bushes was small, we would again expect her to know the dog's location, this time through inference: By bringing to bear the physical knowledge that a large object cannot become fully hidden behind a smaller one, she could infer that her dog must be hidden behind the other, larger bush. Thus, in both cases, we would expect the girl to search the correct location for her dog, based on the information available to her directly through perception or indirectly though inference.

When reasoning about an agent's actions in a scene, can infants also take into account what information might be available to her through perception or inference? Over the past ten years, evidence has steadily accumulated that infants in the first year of life are able to track what information agents can gather through perception (for a review, see Baillargeon et al., 2016). For example, infants aged 6 months and older realize that an agent will be able to see an event and will gain information about it if she is present when it occurs, if she is looking toward it, and if no obstacle blocks her view of it; conversely, the agent will be unable to see the event, and hence will lack information about it, if any of these conditions are not met (Kim \& Song, 2015; Liszkowski et al., 2008; Luo \& Baillargeon, 2007; Luo \& Johnson, 2009; Meristo \& Surian, 2013; Repacholi et al., 2008; Scott \& Baillargeon, 2009; Ting et al., 2019). 
Are young infants also able to track what information agents can garner through inference, by bringing to bear general knowledge about objects? As best we know, no investigation has yet addressed this issue. At first glance, it might appear that a key impediment to doing so would be that young infants possess little general knowledge about objects. However, evidence from the physical-reasoning literature indicates that infants begin acquiring physical rules about occlusion, containment, support, collision, and other events in the first months of life (Aguiar \& Baillargeon, 2002; Hespos \& Baillargeon, 2008; Káldy \& Leslie, 2005; Kotovsky \& Baillargeon, 1998; Luo et al., 2009; Wang \& Onishi, 2017; Wang et al., 2004; Wilcox, 1999). The present research built on these findings to ask the following question: Would 5-month-old infants expect an agent to correctly infer the location of an object hidden in her absence, via the application of relevant physical knowledge?

Not surprisingly, different accounts of infants' ability to reason about others' actions offered different answers to this question. Whereas two-system accounts suggested that infants would be unable to track information the agent could attain only through an inference from relevant physical knowledge, one-system accounts suggested that infants would be able to do so, at least under optimal conditions. In the following sections, we first describe these accounts and then introduce the present research.

\subsection{Different Accounts}

There is currently a heated debate in psychology over how best to characterize the development of children's ability to reason about agents' actions (this ability is variously referred to as psychological reasoning, theory of mind, mindreading, and mentalizing). According to twosystem accounts (Apperly \& Butterfill, 2009; Butterfill, \& Apperly, 2013; Low \& Watts, 2013; Low et al., 2014, 2016), two distinct cognitive systems underlie this development. The early- 
developing system, which emerges in infancy, is automatic, fast, and inflexible, whereas the latedeveloping system, which emerges around 4 years of age, is non-automatic, slow, and flexible. A key strength of the early-developing system is its ability to track what objects agents "register" and to use these registrations to predict their actions. For example, if an agent sees a toy being hidden in one of two locations, the early-developing system can predict, by considering where she last registered the toy, that she will search for it in the correct location. Beyond this ability, however, the early-developing system suffers from a number of "signature limits" that distinguish its minimal capacity for psychological reasoning from the more advanced capacity of the latedeveloping system. Of particular relevance to the present research is the signature limit of cognitive encapsulation: Because the early-developing system is "largely automatic and independent of central cognitive resources" (Low et al., 2016, p. 185), it is encapsulated from the rest of cognition. This makes it fast and efficient, but also inflexible and sharply limited in its ability to integrate inputs from other cognitive processes (e.g., physical reasoning and language) when reasoning about agents' actions (Butterfill \& Apperly, 2013).

In contrast to two-system accounts, one-system accounts assume that a single system underlies psychological reasoning from infancy to adulthood (Baillargeon et al., 2016; Carruthers, 2016; Hyde et al., 2018; Leslie et al., 2004; Scott et al., in press). Although significant developments take place in this system with age and experience, they are largely quantitative in nature: Infants' psychological reasoning is qualitatively similar to that of older children and adults, albeit less well-developed. One-system accounts thus dispute the various signature limits proposed by two-system accounts, including the claim that early psychological reasoning is inflexible and cognitively encapsulated. From a young age, infants' burgeoning psychological-reasoning system is thought to be capable of integrating inputs from other cognitive processes, provided task 
demands do not overwhelm infants' limited information-processing resources (Scott et al., in press).

\subsection{The Present Research}

Would 5-month-old infants expect an agent who was absent while a toy was hidden to still be able to determine its location, if this location could be inferred by bringing to bear relevant physical knowledge? To find out, we took advantage of the prior finding that by 4 months of age, most infants have acquired the physical rule that wide objects can fit into wide but not narrow containers (Wang et al, 2004). In a preference task adapted from Woodward (1998), infants first received familiarization trials in which an agent consistently preferred a wide toy, which jingled when shaken, over a narrow toy, which did not. In the test trial, while the agent was absent, an experimenter hid the wide toy in a wide box and the narrow toy in a narrow box. Of interest was whether infants would expect the agent, when she returned, to correctly infer that her preferred toy was in the wide box, even though she had not witnessed the hiding of the toys.

As should be clear from the previous section, two-system and one-system accounts offered different predictions for infants' responses. According to two-system accounts, the earlydeveloping system should be able to track information the agent registered though perceptionbut it should be unable, due to its cognitive encapsulation, to track information she could attain only through inference, by bringing to bear relevant physical knowledge. In other words, the encapsulated early-developing system should be unable to integrate physical knowledge into its reasoning about what information was available to the agent about the wide toy's location. Infants

should thus hold no particular expectation about which box she would choose, and they should look equally whether she reached for the wide or the narrow box.

In contrast, one-system accounts predicted that infants would be able to integrate physical 
knowledge into their reasoning about what information was available to the agent about the wide toy's location, provided task demands did not overwhelm infants' limited information-processing resources. Specifically, infants should assume that the agent (a) would share the physical knowledge that wide objects can fit only into wide containers and hence (b) would infer that the wide toy must be hidden in the wide box. Infants should thus expect the agent to reach for the wide box, and they should find it unexpected when she reached for the narrow box instead.

Which of the two preceding predictions was more likely to be correct? Findings from two prior reports, in particular, seemed to us to provide tentative support for the prediction from onesystem accounts. In the first report, 18-month-olds attributed to an agent a false inference based on a statistical regularity about objects (similar objects are more likely to share non-obvious properties than are dissimilar objects; Welder \& Graham, 2001) that happened to not hold true in the experimental situation (Scott et al., 2010; for a replication in traditional non-Western societies, see Barrett et al., 2013). In a familiarization trial, Agent-1 sat at a side window in a puppet-stage apparatus and faced a red toy with silver stars. Two additional toys stood at the rear of the apparatus: One was visually identical to Agent-1's toy (red test toy) and the other was green with yellow stripes (green test toy). Agent-1 shook each toy in turn, thereby revealing that her toy and the green test toy rattled when shaken but the red test toy did not. In the test trial, Agent-2 now sat behind the two test toys. Agent-1 shook her toy and said, "Can you do it?" Agent-2 then grasped either the red or green test toy and paused. Infants looked significantly longer if she selected the green as opposed to the red test toy, suggesting that they expected her (a) to share the intuition that similar objects are more likely than dissimilar objects to have the same non-obvious properties and hence (b) to falsely infer that the red test toy rattled. If 18-month-olds could attribute to an agent a false inference based on a statistical regularity about objects' non-obvious properties, it seemed 
possible that 5-month-olds could attribute to an agent a true inference based on a physical rule about containment events. ${ }^{1}$

The second report hinted at some degree of cognitive integration in the first half-year of life between infants' psychological reasoning and another cognitive process, language (Vouloumanos et al., 2014). In the report, 6-month-olds were tested with a preference task that made use of the linguistic knowledge that words are linguistic conventions that are shared by members of the same speech community (Henderson \& Woodward, 2012). In the familiarization trials, Agent-1 consistently selected object-A over object-B, indicating that she preferred it. In the test trial, Agent-1 could no longer reach the objects (she peered at them through a small window) and was joined by Agent-2, who could reach both objects. Agent-1 looked at Agent-2 and said, “Koba, koba!” Infants expected Agent-2 to pick up object-A, suggesting that they (a) guessed that this novel word referred to Agent-1's preferred object and (b) expected Agent-2 to already know the meaning of this word and hence to give object-A to Agent-1. If 6-month-olds could integrate language into their psychological reasoning, it seemed possible that 5-month-olds could integrate physical knowledge as well.

\footnotetext{
${ }^{1}$ Other experiments have shown that infants in the second year of life can attribute to agents inferences based on local patterns established through simple repetition (Buttelmann et al., 2014; Scott \& Baillargeon, 2009; Song \& Baillargeon, 2008; Surian et al., 2007). For example, after an agent was shown that three identical boxes, introduced separately, each contained a block, 18month-olds expected the agent to falsely assume that a fourth identical box also contained a block (infants knew this box in fact contained a spoon; Buttelmann et al., 2014). Similarly, after an agent saw a desired object being hidden behind one screen four times, 13-month-olds expected the agent, when returning to the scene after the object was already hidden, to falsely infer that it had been hidden in the same location as before (infants knew the object had in fact been hidden behind a different screen; Surian et al., 2007). In each case, infants thus attributed to the agent the inference that the latest event in the scene had followed the same pattern as the preceding events. In contrast to inferences based on general knowledge about objects, which depend on the integration of infants' psychological reasoning with other cognitive processes, these inferences based on local patterns require little or no cognitive integration and, as such, are compatible with both two-system and one-system accounts of early psychological reasoning.
} 


\section{Design}

Infants were assigned to one of four conditions $(N=96$, with 24 per condition): inference, ignorance, no-preference, and perception. Each infant sat on a parent's lap facing a puppet-stage apparatus and received four familiarization trials and one test trial. Each trial had a computercontrolled initial phase followed by an infant-controlled final phase. During the initial phase, infants saw the scripted events appropriate for the trial, ending with a paused scene; during the final phase, infants watched this paused scene until the trial ended.

At the start of the initial phase in the first two familiarization trials of the inference condition (Fig. 1), a female agent sat at a window in the back wall of the apparatus, behind two rectangular mats. In front of each mat was a toy that consisted of a tall handle attached to a wide (left toy) or narrow (right toy) rectangular base; the toys' bases and the lower halves of their handles differed in color and pattern, but the upper halves of their handles were identical and each consisted of a thin yellow rod with a bright pink ornament at the top. As the agent watched, an experimenter's gloved hand entered the apparatus through a curtained window in the right wall. The hand grasped the handle of the nearer toy (about $5 \mathrm{~cm}$ below the pompom), shook it four times, and placed it on its mat; next, the hand performed the same actions on the farther toy. The wide toy jingled when shaken, but the narrow toy did not. After the hand completed its actions and left, the agent grasped the handle of the wide toy and shook it four times, causing it to jingle. Next, she returned the wide toy to its mat and then paused, with her hands on the apparatus floor. During the final phase, infants watched this paused scene until the trial ended. The last two familiarization trials were identical except that round mats were used and the toys' locations were switched; as before, the gloved hand acted on the nearer toy first. The familiarization trials thus served to establish that the agent preferred the wide toy, presumably because it jingled when shaken (Bahrick 
et al., 2002; Perone et al., 2008), and that she consistently reached for it irrespective of where it was placed in the apparatus.

During the initial phase of the test trial (Fig. 2), the agent was at first absent: Her window was closed. On the apparatus floor, instead of two mats, there were now two tall boxes, open at the top, that differed in color and pattern as well as width: The box on the left was sufficiently wide to hold either the wide or the narrow toy, whereas the box on the right was narrow and could hold only the narrow toy. The wide toy stood in front of the wide box, and the narrow toy stood in front of the narrow box. As in the familiarization trials, the gloved hand entered the apparatus, shook the nearer toy, placed it in its box, and then repeated these actions with the farther toy. Because both boxes were equally tall, only the identical upper portions of their handles protruded from them. After the hand completed its actions and left, the agent opened her window, grasped the handle of either the wide (wide-toy event) or the narrow (narrow-toy event) toy, and paused. During the final phase of the trial, infants watched this paused scene until the trial ended.

We reasoned that finding that infants looked significantly longer if shown the narrow-toy as opposed to the wide-toy event would suggest that they (a) attributed to the agent a preference for the wide, jingling toy over the narrow, silent toy during the familiarization trials, (b) expected her to continue acting on this preference in the test trial, (c) realized that although she was absent when her preferred toy was hidden, she could infer its location by bringing to bear the physical knowledge that wide objects can fit only into wide containers, and hence (d) expected her to reach for the handle protruding from the wide box and found it unexpected when she reached for the handle protruding from the narrow box instead.

Additional infants were tested in three conditions designed to support this interpretation of positive findings in the inference condition. The ignorance condition served to rule out one 
alternative interpretation of such findings: Perhaps infants in the inference condition acted egocentrically and attributed to the agent their own knowledge of the wide toy's location. This possibility seemed unlikely because 6-month-olds have been shown in a variety of tasks to nonegocentrically keep track of what others can see or have seen (Kim \& Song, 2015; Luo \& Johnson, 2009; Southgate \& Vernetti, 2014). Nevertheless, to rule out this possibility, infants in this condition were tested using the same procedure as in the inference condition with one exception: In the test events, the narrow box was replaced with another wide box of the same color and pattern as the narrow box. If infants simply attributed to the agent their own knowledge of where the wide toy was hidden, results should be similar to those of the inference condition. On the other hand, if infants realized, non-egocentrically, that the agent had to be ignorant about the wide toy's location because there was no basis for her to infer this location (both boxes were wide enough to accommodate the wide toy, and the handles protruding from them were identical), then infants should look equally whether they were shown the narrow-toy or the wide-toy test event.

The no-preference condition addressed another alternative interpretation of positive findings in the inference condition: Perhaps infants looked longer when the agent reached for the narrow box simply because they had a baseline preference for that box. To rule out this possibility, infants in this condition were tested using the same procedure as in the inference condition except that the test events were modified in two ways: The agent was present throughout each event and watched as the hand shook each toy and placed it in its box, and the wide toy no longer jingled when shaken, as though broken. If infants simply had a baseline preference for the narrow box, results should be the same as in the inference condition. On the other hand, if infants were reasoning about which toy the agent would reach for, then results might differ from those in the inference condition. As the wide toy no longer jingled, infants might expect the agent's preference 
for that toy to dissipate, leaving them with no particular expectation about which toy she would select. Infants should thus look equally at the narrow- and wide-toy test events.

Finally, the perception condition was designed to support positive findings in the inference condition by showing that similar results were found when the agent directly observed the hiding of the two toys in the test events. Infants in this condition were tested using the same procedure as in the ignorance condition, with one exception: The agent was present and watched as the hand shook each toy and placed it in its box. Based on prior findings that infants aged 6 months and older keep track of what events others have seen (Hyde et al., 2018; Kampis et al., 2015; Kovács et al., 2010; Liszkowski et al., 2008; Luo \& Johnson, 2011; Meristo \& Surian, 2013; Southgate \& Vernetti, 2014; Ting et al., 2019), infants should expect the agent to reach for the box that held her preferred toy, and they should find it unexpected when she reached for the box that held the other toy instead. As in the inference condition, infants should thus look significantly longer if shown the narrow-toy as opposed to the wide-toy event.

In short, finding positive results in the inference and perception conditions but negative results in the ignorance and no-preference conditions would indicate that from a young age, infants track not only what information agents can gather though perception but also what information they can gather through inference, by bringing to bear relevant general knowledge. Such results would cast doubt on the claim by two-system accounts that early psychological reasoning is inflexible and cognitively encapsulated and, by the same token, would support one-system accounts.

\section{Method}

\subsection{Power analysis}

Luo and Baillargeon (2007) compared infants' ability to keep track of what objects an agent 
could and could not see in the context of a preference task, using live events and a $2 \times 2$ betweensubject design; the Condition $\times$ Event effect size $\left(\eta_{p}{ }^{2}\right)$ they obtained was 0.163 . Using this value, we conducted an a-priori power analysis with $G^{*}$ Power (Faul, Erdfelder, Lang, \& Buchner, 2007) to estimate the appropriate sample size for our experiment. Although our overall design was a $4 \times$ 2 between-subject design, our analysis plan also included comparing the inference condition to the other conditions in separate $2 \times 2$ ANOVAS. With power set at .80 and alpha set at .05 , the minimum number of infants required per cell (i.e., per combination of condition and event) for these comparisons was between ten and eleven infants. In line with this estimate, we tested 12 infants per cell, for a total of 24 per condition and 96 overall.

\subsection{Participants}

Participants were 96 healthy term 5-month-old infants, 46 male (range $=4$ months, 5 days to 7 months, 4 days, $M=5$ months, 13 days). Another 14 infants were tested but excluded from the analyses. Of these, 12 (distributed across the four conditions) were fussy (8), drowsy (2), or distracted (e.g., by straining); the other 2 infants looked over 3 standard deviations above the mean in the test trial ( 1 in the inference condition and 1 in the perception condition; both infants saw the narrow-toy test event). Twenty-four infants were randomly assigned to each of the four conditions; in the inference, ignorance, and perception conditions, 12 infants saw the narrow-toy test event and 12 saw the wide-toy test event; in the no-preference condition, the corresponding numbers were 13 and 11.

Infants' names were obtained from a university-maintained database of parents interested in participating in child-development research. Parents were offered a small gift (e.g., a children's book) and travel reimbursement but were otherwise not compensated for their participation. Each infant's parent gave written informed consent, and the protocol was approved by the Institutional 
Review Board at the University of Illinois at Urbana-Champaign.

\subsection{Apparatus and Stimuli}

The apparatus consisted of a brightly lit display booth $(201 \mathrm{~cm}$ high $\times 102 \mathrm{~cm}$ wide $\times 58$ $\mathrm{cm}$ deep) with a large opening $(55 \mathrm{~cm} \times 95 \mathrm{~cm})$ in its front wall; between trials, a supervisor lowered a curtain in front of this opening. Inside the apparatus, the side and back walls were white, and the floor was covered with a lightly patterned adhesive paper.

Two female assistants served as the agent and the experimenter. The agent wore a grey shirt and sat at a window $(50 \times 50 \mathrm{~cm})$ in the back wall of the apparatus; this window was located $7 \mathrm{~cm}$ from the right wall and could be closed by two small white doors. The experimenter wore a white shirt and a long white glove on her left hand and arm, and she knelt at a window $(51 \times 38$ $\mathrm{cm})$ in the right wall that was filled with a white fringe curtain; she introduced her gloved hand into the apparatus through the curtain. Behind the two assistants, white ceiling-to-floor curtains hid the testing room from infants' view. During the trials, the agent followed the gloved hand's actions, looked at the objects she acted on, and otherwise looked at a neutral point on the apparatus floor; she never made eye contact with the infant.

The wide toy had a rectangular base $(4 \times 15 \times 4 \mathrm{~cm})$ that was covered with red adhesive paper and decorated with various colorful stickers. At the back of the base, out of infants' view, was a Velcro strip with four metal bells that jingled when shaken (this strip was removed prior to the test trial in the no-preference condition). A spool (5 cm tall and $4 \mathrm{~cm}$ in diameter), also covered with red adhesive paper, was centered on the top of the base. A thin rod $(25 \mathrm{~cm}$ tall and $0.5 \mathrm{~cm}$ in diameter) protruded from the center of the spool; its lower half was covered with red adhesive paper and its top half with yellow adhesive paper. At the top of the rod was a section of a bright pink kush ball ( $2 \mathrm{~cm}$ tall and $2.5 \mathrm{~cm}$ in diameter), which helped make the rod more noticeable for 
infants. The narrow toy was similar, with the following exceptions: Its base was narrower $(4 \mathrm{~cm}$ wide); it had no bells; its base, its spool, and the lower half of its handle were covered with a dark green adhesive paper; and the front of its base was decorated with a large gold star. The total height of each toy was $36 \mathrm{~cm}$.

Strictly speaking, our experimental design did not require the use of handles for the toys, as the agent and the gloved hand could have acted directly on the toys' bases or on the boxes. There were several reasons for using handles. First, when the toys were in the boxes, their protruding handles ensured that they remained partly visible to infants, thereby reducing information-processing demands (Hauf et al., 2012; Hespos \& Baillargeon, 2006; Lin et al., 2021). Second, having the agent reach for a handle rather than for a box in the test trial prevented any confusion about whether she was now interested in the box as opposed to the toy. Finally, because the handles of the two toys were identical, the agent's actions were highly similar in the paused scenes at the end of all test events (i.e., she always grasped an identical handle and paused).

The rectangular mats used in the first two familiarization trials $(0.5 \times 19 \times 9.5 \mathrm{~cm})$ were covered with a white, blue, and green plaid-patterned adhesive paper, and the round mats used in the last two familiarization trials $(0.2 \mathrm{~cm}$ tall and $19 \mathrm{~cm}$ in diameter $)$ were covered with a brown wood-patterned adhesive paper. Across conditions, three tall boxes (each $26 \mathrm{~cm}$ tall $\times 9.5 \mathrm{~cm}$ deep) were used in the test trial. Two of the boxes were wide $(21 \mathrm{~cm})$; one was covered with a light green marble-pattern adhesive paper and the other with a light blue adhesive paper decorated with a subtle white dot pattern; the last box was narrow $(9 \mathrm{~cm})$ and was covered with the same light blue adhesive paper. At the start of the trials, the toys stood centered $5 \mathrm{~cm}$ in front of the rectangular mats or boxes, which themselves stood $12 \mathrm{~cm}$ apart, centered in front of and $17 \mathrm{~cm}$ from the agent's window; the corresponding values for the rounded mats were $0.5,12$, and $12 \mathrm{~cm}$. 
During each testing session, two cameras captured images of the infant and events; the two images were combined, projected onto a monitor located behind the apparatus, and checked by the supervisor to confirm that the events followed the prescribed scripts. Recorded sessions were also checked off-line for experimenter accuracy.

\subsection{Procedure}

Each infant sat on a parent's lap centered in front of the apparatus; parents were instructed to remain silent and neutral throughout the session and to close their eyes during the test trial. Each infant's looking behavior was monitored by two naïve observers hidden behind cloth-covered panels on either side of the apparatus; looking times during the initial and final phases of each trial were computed separately, using the primary observer's responses. Interobserver agreement was calculated for each trial by determining the proportion of $100-\mathrm{ms}$ intervals in which the two observers agreed during the final phase of the trial. Agreement was calculated for 92/96 infants (only one observer was present for the other infants) and averaged 92\% per trial per infant.

Each trial began with a paused pretrial that ended when the infant had cumulated $2 \mathrm{~s}$ of looking, to allow the infant to orient to the apparatus before the trial proper began. The durations of the initial phases in the familiarization (25-s) and test (23-s) trials were fixed and computercontrolled; the two assistants' actions followed a precise second-by-second script, and a metronome beat softly once per second to help them adhere to the script. Infants were highly attentive during these initial phases; across conditions, they looked, on average, for $97 \%$ of the initial phase in each familiarization trial and for $97 \%$ of the initial phase in the test trial. The final phases of the familiarization trials ended when infants (a) looked away for 2 consecutive seconds after having looked for at least 5 cumulative seconds or (b) looked for a maximum of 60 cumulative seconds. Such criteria are commonly used in infancy research and give infants ample opportunity 
to become familiar with the events shown. The final phase of the test trial ended when infants (a) looked away for 0.5 seconds after having looked for at least 8 seconds or (b) looked for a maximum of 30 seconds. The 8-s minimal value was chosen to give our young participants sufficient time to process the agent's toy selection before the trial could end. In addition, a 0.5-s look-away criterion was used because the agent paused at a different point in the test trial than she did in the familiarization trials. In the latter trials, she shook the wide toy four times and then paused; in the test trial, however, she grasped the handle of one of the toys and then paused. Infants therefore tended to look back at the paused scene because their attention was recaptured as they waited for the agent to shake the toy, as she had done before. By using a short, 0.5-s look-away criterion, we could assess infants' initial response to the agent's selection of the wide or narrow toy (for other studies using a 0.5-s look-away criterion, see e.g., Scott \& Baillargeon, 2013; Stavans \& Baillargeon, 2018). ${ }^{2}$

To reduce positive skewness, all looking times were log-transformed, and analyses were

\footnotetext{
${ }^{2}$ A concern some readers might have about our procedure was that it used a variant of the violation-of-expectation method that is commonly used to study early psychological reasoning (e.g., infants saw a single test event, which was shown only once, followed by a paused scene; Luo \& Baillargeon, 2007; Onishi \& Baillargeon, 2005; Scott et al., 2010; Surian et al., 2007; Vouloumanos et al., 2014). By contrast, when the violation-of-expectation method was first introduced several decades ago to study early physical reasoning, infants often saw two different test events on alternate trials, and in each trial the event was repeated continuously until the trial ended (Aguiar \& Baillargeon, 2002; Baillargeon et al., 1985; Baillargeon \& DeVos, 1991; Kotovsky \& Baillargeon, 1998; Needham \& Baillargeon, 1993). Because the present research examined the integration of psychological and physical reasoning in young infants, this juxtaposition might give rise to the worry that infants could fail in the inference condition, not because they were unable to track information gathered through inference, but because the procedure chosen was less than optimal for revealing their physical-reasoning capacity. In recent years, however, several reports have used a single-event, paused-scene procedure to study various facets of infants' physical reasoning, with no ill effects (Lin et al., 2021; Stavans \& Baillargeon, 2018; Stavans et al., 2019; Wang \& Goldman, 2016; Wang et al., 2016). There was thus little apriori reason for concern, and the positive results of the inference condition confirmed that the procedure selected was appropriate for the present research.
} 
conducted on the log-transformed data (Csibra et al., 2016). For ease of communication, however, raw looking times are provided in the text and figures. Preliminary analyses of the test data revealed no significant interaction of condition and event with the infant's sex, $F(3,80)=1.00, p$ $=.396$; the data were therefore collapsed across this factor in subsequent analyses. The data are available via the Open Science Framework and can be accessed at https://osf.io/u9gda/?view_only=92c4b3ac3d9949f084b0b623406c9b53.

\section{Results}

Infants' looking times during the final phases of the four familiarization trials were averaged and analyzed by means of a $4 \times 2$ analysis of variance (ANOVA) with condition (inference, ignorance, no-preference, perception) and test event (wide-toy, narrow-toy) as between-subject factors. The Condition $\times$ Event interaction was not significant, $F(3,88)=0.51, p$ $=.674, \eta_{p}^{2}=.017$, nor was either main effect, both $p \mathrm{~s}>.692$, suggesting that infants tended to look equally during the familiarization trials (inference: $M=26.25, S D=13.16$; ignorance: $M=$ 26.47, $S D=11.19 ;$ no-preference: $M=26.33, S D=13.59$; perception: $M=23.83, S D=9.61)$.

Infants' looking times during the final phase of the test trial (Fig. 3) were analyzed in the same manner as above. The analysis yielded a significant main effect of event, $F(1,88)=5.49, p$ $=.021$, as well as a significant Condition $\times$ Event interaction, $F(3,88)=6.18, p=.0007, \eta_{p}{ }^{2}=$ .174. To examine this interaction, we conducted four planned comparisons, one per condition (with a Bonferroni-adjusted $\alpha$-level of $.05 / 4=0.0125)$. Infants in the inference condition looked significantly longer if shown the narrow-toy event $(M=19.18, S D=8.41)$ as opposed to the widetoy event $(M=10.41, S D=2.43), F(1,88)=15.25, p=.0002, d=1.462$; infants in the ignorance condition looked equally at the narrow-toy $(M=13.21, S D=7.16)$ and wide-toy $(M=14.78, S D$ $=6.75)$ events, $F(1,88)=0.93, p=.338, d=-0.309$; infants in the no-preference condition again 
looked equally at the narrow-toy $(M=11.27, S D=3.16)$ and wide-toy $(M=12.78, S D=3.51)$ events, $F(1,88)=0.82, p=.367, d=-0.468$; and infants in the perception condition looked significantly longer if shown the narrow-toy event $(M=17.54, S D=4.45)$ as opposed to the widetoy event $(M=12.16, S D=2.97), F(1,88)=7.06, p=.009, d=1.438$. Non-parametric Wilcoxon rank-sum tests (two-sided) confirmed the results of the inference $(Z=2.80, p=.005)$, ignorance $(Z=-1.13, p=.260)$, no-preference $(Z=0.96, p=.339$, and perception $(Z=3.06, p=.002)$ conditions.

Finally, $2 \times 2$ ANOVAS indicated that test responses in the inference condition differed significantly from those in the ignorance condition (Condition $\times$ Event interaction: $F(1,44)=8.46$, $\left.p=.006, \eta_{p}^{2}=.161\right)$ and the no-preference condition $\left(F(1,44)=12.71, p=.0009, \eta_{p}^{2}=.224\right)$, but not the perception condition $\left(F(1,44)=0.89, p=.352, \eta_{p}^{2}=.020\right)$. In line with prior findings, test responses in the perception condition also differed significantly from those in the ignorance condition $\left(F(1,44)=6.00, p=.018, \eta_{p}^{2}=.120\right)$ and the no-preference condition $(F(1,44)=10.58$, $\left.p=.002, \eta_{p}^{2}=.194\right)$.

\section{Discussion}

In the present research, 5-month-olds received familiarization trials in which an agent first saw an experimenter shake a wide toy, which jingled when shaken, and a narrow toy, which did not; the agent then grasped the wide toy and shook it. In the test trial, while the agent was absent, the experimenter shook each toy as before and hid the wide toy in a wide box and the narrow toy in a narrow box (inference condition). When the agent returned, infants looked significantly longer if she reached for the narrow as opposed to the wide toy, suggesting that they (a) attributed to the agent a preference for the wide, jingling toy during the familiarization trials, (b) assumed that the agent shared the knowledge that wide objects can fit only into wide containers, (c) expected the 
agent to apply this knowledge in the test trial to infer which box held the wide toy, and hence (d) found it unexpected when she reached for the narrow toy instead. This effect was eliminated when the two boxes used in the test trial were both wide, leaving the agent with no basis for inferring the wide toy's location (ignorance condition). Negative results were also found when the agent witnessed the hiding of the toys into the wide and narrow boxes, but the wide toy no longer jingled when shaken by the experimenter, causing the agent's preference for the toy to dissipate (nopreference condition). Finally, positive results were again found when the agent witnessed the hiding of the toys into the two wide boxes and thus saw where the experimenter hid her preferred toy (perception condition).

Together, these results rule out several low-level interpretations of the positive results in the inference condition, such as that infants attributed to the agent their own knowledge of the wide toy's location (had that been the case, positive results would have also been found in the ignorance condition), that infants simply had a baseline preference for the narrow box (positive results would have also been found in the no-preference condition), or that infants themselves could remember the location of the agent's preferred toy only when the two boxes differed in width (negative results would have been found in the perception condition). In addition, our design rules out low-level hypotheses that similar results were found whenever the agent was absent at the start of the test trial (results were positive in the inference condition but negative in the ignorance condition), whenever the agent was present at the start of the trial (results were positive in the perception condition but negative in the no-preference condition), whenever the two boxes differed in width (results were positive in the inference condition but negative in the no-preference condition), or whenever the two boxes were identical in width (results were positive in the perception condition but negative in the ignorance condition). 
Our results provide converging evidence for prior findings in early psychological reasoning. In particular, the results of the perception condition confirm that young infants who see an agent consistently choose one object over another attribute to the agent a preference for that object and expect the agent to continue acting on this preference when the objects' locations are changed in her presence (Baillargeon et al., 2016; Luo, 2011; Luo \& Baillargeon, 2005; Spaepen \& Spelke, 2007; Woodward, 1998, 1999). Moreover, the results of the ignorance condition provide additional evidence that young infants are not egocentric: When an agent's representation of a scene is less complete than their own, they use the agent's incomplete representation to interpret, predict, evaluate, and respond to her actions (Kim \& Song, 2015; Liszkowski et al., 2008; Luo \& Baillargeon, 2007; Luo \& Johnson, 2009; Meristo \& Surian, 2013; Ting et al., 2019).

Our results also extend prior research in two significant ways. First, they expand our understanding of early epistemic reasoning (i.e., reasoning about knowledge and ignorance states). Specifically, they reveal that when determining what an agent knows about a scene, 5-month-old infants take into account not only what information she can obtain through direct perception but also what information she can garner through inference, via the application of relevant physical rules; they then expect her to act in a manner consistent with her knowledge, however it was attained. From this perspective, our findings add to the evidence that early psychological reasoning is guided by a principle of rationality and its corollaries of consistency (agents act in a manner consistent with their mental states) and efficiency (agents expend as little effort as possible to achieve their goals) (Baillargeon et al., 2016; Gergely \& Csibra, 2003; Ting \& Baillargeon, 2020; Ting et al., in press). In the inference condition, infants expected the agent to act in a manner consistent with her motivational state (she preferred the wide, jingling toy) and her epistemic state (she could infer that it was in the wide box), and they detected a consistency violation when she 
reached for the narrow toy instead.

Second, our results bear on the ongoing debate between two-system and one-system accounts of early psychological reasoning. As was discussed in the Introduction, two-system accounts assume that infants' primitive early-developing system is sufficient to track information registered through direct perception but is too inflexible and encapsulated to track information supplied by other cognitive processes (Apperly \& Butterfill, 2009; Butterfill \& Apperly, 2013; Low \& Watts, 2013; Low et al., 2014, 2016). According to one-system accounts, in contrast, infants' psychological-reasoning system is neither inflexible nor cognitively encapsulated and can integrate inputs from other cognitive processes, at least under optimal conditions (Baillargeon et al., 2016; Carruthers, 2016; Hyde et al., 2018; Leslie et al., 2005; Scott et al., in press). Our finding that infants as young as 5 months of age were able to attribute to an agent knowledge she could attain only by applying relevant physical rules to the scene thus provides new evidence for onesystem accounts.

In a recent review, Scott et al. (in press) presented evidence of substantial cognitive integration in psychological reasoning in the second year of life: Infants integrated inputs from several different cognitive processes, including language, ostensive cues, categorization, and sociomoral reasoning, when reasoning about others' motivational and epistemic states (Choi \& Luo, 2015; Egyed et al., 2013; Forgács et al., 2020; Jin \& Song, 2017; Jin et al., 2019; Martin et al., 2012; Scott et al., 2010; Spaepen \& Spelke, 2007). The present findings, together with those of Vouloumanos et al. (2014) described in the Introduction, thus extend this evidence by showing that infants' psychological reasoning already shows some degree of cognitive integration with physical-reasoning and language processes in the first half-year of life.

Future research can build on our findings in several directions. First, it will be important to 
confirm our findings with other physical rules. For example, the rule that tall objects can become fully hidden in tall but not short containers is typically acquired by 7 months of age (Hespos \& Baillargeon, 2001); would 8-month-olds expect an agent who was searching for a tall object hidden in her absence to approach a tall as opposed to a short container? Second, it would be useful to ascertain whether infants expect an agent to draw physical inferences from what she sees even when these inferences no longer apply. To illustrate, imagine that in the inference condition was modified so that in the test trial, the red toy was now as narrow as the green toy. When the agent arrived and saw the handles protruding from the wide and narrow boxes, would infants expect her (a) to falsely assume, based on the familiarization trials, that a wide and a narrow toy were hidden in the boxes and (b) to falsely infer that her preferred wide toy must be in the wide box? Positive results would not only add to the evidence of false-belief understanding in young infants (Hyde et al., 2018; Kampis et al., 2015; Kovács et al., 2010; Southgate \& Vernetti, 2014), but would make clear that infants expect an agent to draw physical inferences from her representation of a scene even when this representation is faulty, making these inferences invalid.

\section{Conclusion}

In the present research, 5-month-old infants watched an agent search for a wide toy that had been hidden in her absence in one of two boxes. Infants always knew in which box the toy was hidden; they expected the agent to correctly infer the toy's location when only one of the boxes was wide enough to hide the toy, but to be ignorant about this location when both boxes were wide enough to hide the toy. These results indicate that when forming expectations about an agent's actions in a scene, infants consider not only what she sees, but also what physical inferences she can derive from what she sees. As such, our results provide new evidence for onesystem accounts and for the claim that far from being inflexible and encapsulated, infants' 
psychological reasoning already show signs of integration with other cognitive processes in the first half-year of life. 


\section{References}

Aguiar, A., \& Baillargeon, R. (2002). Developments in young infants' reasoning about occluded objects. Cognitive Psychology, 45, 267-336.

Apperly, I. A., \& Butterfill, S. A. (2009). Do humans have two systems to track beliefs and belieflike states? Psychological Review, 116, 953-970.

Bahrick, L. E., Gogate, L. J., \& Ruiz, I. (2002). Attention and memory for faces and actions in infancy: The salience of actions over faces in dynamic events. Child Development, 73(6), $1629-1643$.

Baillargeon, R., \& DeVos, J. (1991). Object permanence in young infants: Further evidence. Child Development, 62, 1227-1246.

Baillargeon, R., Scott, R. M., \& Bian, L. (2016). Psychological reasoning in infancy. Annual Review of Psychology, 67, 159-186.

Baillargeon, R., Spelke, E. S., \& Wasserman, S. (1985). Object permanence in five-month-old infants. Cognition, 20, 191-208.

Barrett, H. C., Broesch, T., Scott, R. M., He, Z., Baillargeon, R., Wu, D., ... \& Laurence, S. (2013). Early false-belief understanding in traditional non-Western societies. Proceedings of the Royal Society of London B: Biological Sciences, 280, 20122654.

Buttelmann, D., Over, H., Carpenter, M., \& Tomasello, M. (2014). Eighteen-month-olds understand false beliefs in an unexpected-contents task. Journal of Experimental Child Psychology, 119, 120-26

Butterfill, S., \& Apperly, I. A. (2013). How to construct a minimal theory of mind. Mind and Language, 28, 606-637.

Carruthers, P. (2016). Two systems for mindreading? Review of Philosophy and Psychology, 7 , 
141-162.

Choi, Y. J., \& Luo, Y. (2015). 13-month-olds' understanding of social interactions. Psychological Science, 26, 274-283.

Csibra, G., Hernik, M., Mascaro, O., Tatone, D., \& Lengyel, M. (2016). Statistical treatment of looking-time data. Developmental Psychology, 52(4), 521-536.

Egyed, K., Király, I., \& Gergely, G. (2013). Communicating shared knowledge in infancy. Psychological Science, 24, 1348-1353.

Faul, F., Erdfelder, E., Lang, A. G., \& Buchner, A. (2007). G* Power 3: A flexible statistical power analysis program for the social, behavioral, and biomedical sciences. Behavior Research Methods, 39(2), 175-191.

Forgács, B., Gervain, J., Parise, E., Csibra, G., Gergely, G., Baross,J., \& Király, I. (2020). Electrophysiological investigation of infants' understanding of understanding. Developmental Cognitive Neuroscience, 43, 100783.

Gergely, G., \& Csibra, G. (2003). Teleological reasoning in infancy: The naive theory of rational action. Trends in Cognitive Sciences, 7, 287-292.

Hauf, P., Paulus, M., \& Baillargeon, R. (2012). Infants use compression information to infer objects' weights: Examining cognition, exploration, and prospective action in a preferentialreaching task. Child Development, 83, 1978-1995.

Henderson, A. M., \& Woodward, A. L. (2012). Nine-month-old infants generalize object labels, but not object preferences across individuals. Developmental Science, 15, 641-652.

Hespos, S. J., \& Baillargeon, R. (2001). Infants' knowledge about occlusion and containment events: A surprising discrepancy. Psychological Science, 12, 141-147.

Hespos, S. J., \& Baillargeon, R. (2006). Décalage in infants' knowledge about occlusion and 
containment events: Converging evidence from action tasks. Cognition, 99, B31-B41.

Hespos, S. J., \& Baillargeon, R. (2008). Young infants' actions reveal their developing knowledge of support variables: Converging evidence for violation-of-expectation findings. Cognition, $107,304-316$.

Hyde, D. C., Simon, C. E., Ting, F., \& Nikolaeva, J. I. (2018). Functional organization of the temporal-parietal junction for theory of mind in preverbal infants: A near-infrared spectroscopy study. Journal of Neuroscience, 38, 4264-4274.

Jin, K. S., \& Song, H. J. (2017). You changed your mind! Infants interpret a change in word as signaling a change in an agent's goals. Journal of Experimental Child Psychology, 162, 149162.

Jin, K. S., Kim, Y., Song, M., Kim, Y. J., Lee, H., Lee, Y., Cha, M., \& Song, H. J. (2020). Fourteento eighteen-month-old infants use explicit linguistic information to update an agent's false belief. Frontiers in Psychology, 10, 2508.

Káldy, Z., \& Leslie, A. M. (2005). A memory span of one? Object identification in 6.5-monthold infants. Cognition, 97, 153-177.

Kampis, D., Parise, E., Csibra, G., \& Kovács, Á. M. (2015). Neural signatures for sustaining object representations attributed to others in preverbal human infants. Proceedings of the Royal Society B: Biological Sciences, 282, 20151683.

Kim, E. Y., \& Song, H. J. (2015). Six-month-olds actively predict others' goal-directed actions. Cognitive Development, 33, 1-13.

Kotovsky, L., \& Baillargeon, R. (1998). The development of calibration-based reasoning about collision events in young infants. Cognition, 67, 311-351.

Kovács, Á. M., Téglás, E., \& Endress, A. D. (2010). The social sense: Susceptibility to others’ 
beliefs in human infants and adults. Science, 330, 1830-1834.

Leslie, A. M., Friedman, O., \& German, T. P. (2004). Core mechanisms in 'theory of mind'. Trends in Cognitive Sciences, 8, 528-533.

Lin, Y., Li, J., Gertner, Y., Fisher, C. L., \& Baillargeon, R. (2021). How do the object-file and physical-reasoning systems interact? Evidence from priming effects with object arrays or novel labels. Cognitive Psychology, 125, article 101368.

Liszkowski, U., Carpenter, M., \& Tomasello, M. (2008). Twelve-month-olds communicate helpfully and appropriately for knowledgeable and ignorant partners. Cognition, 108, 732-739.

Low, J., Apperly, I. A., Butterfill, S. A., \& Rakoczy, H. (2016). Cognitive architecture of belief reasoning in children and adults: A primer on the two-systems account. Child Development Perspectives, 10, 184-189.

Low, J., Drummond, W., Walmsley, A., \& Wang, B. (2014). Representing how rabbits quack and competitors act: Limits on preschoolers' efficient ability to track perspective. Child Development, $85,1519-1534$.

Low, J., \& Watts, J. (2013). Attributing false beliefs about object identity reveals a signature blind spot in humans' efficient mind-reading system. Psychological Science, 24, 305-311.

Luo, Y. (2011). Three-month-old infants attribute goals to a non-human agent. Developmental Science, 14, 453-460.

Luo, Y., \& Baillargeon, R. (2005). Can a self-propelled box have a goal? Psychological reasoning in 5-month-old infants. Psychological Science, 16, 601-608.

Luo, Y., \& Baillargeon, R. (2007). Do 12.5-month-old infants consider what objects others can see when interpreting their actions? Cognition, 105, 489-512.

Luo, Y., \& Johnson, S. C. (2009). Recognizing the role of perception in action at 6 months. 
Developmental Science, 12, 142-149.

Luo, Y., Kaufman, L., \& Baillargeon, R. (2009). Young infants' reasoning about physical events involving inert and self-propelled objects. Cognitive Psychology, 58, 441-486.

Martin, A., Onishi, K. H., \& Vouloumanos, A. (2012). Understanding the abstract role of speech in communication at 12 months. Cognition, 123, 50-60.

Needham, A., \& Baillargeon, R. (1993). Intuitions about support in 4.5-month-old infants. Cognition, 47, 121-148.

Meristo, M., \& Surian, L. (2013). Do infants detect indirect reciprocity? Cognition, 129, 102-113.

Onishi, K. H., \& Baillargeon, R. (2005). Do 15-month-old infants understand false beliefs? Science, 308, 255-258.

Perone, S., Madole, K. L., Ross-Sheehy, S., Carey, M., \& Oakes, L. M. (2008). The relation between infants' activity with objects and attention to object appearance. Developmental Psychology, 44(5), 1242-1248.

Repacholi, B. M., Meltzoff, A. N., \& Olsen, B. (2008). Infants' understanding of the link between visual perception and emotion: "If she can't see me doing it, she won't get angry." Developmental Psychology, 44, 561-574.

Scott, R. M., \& Baillargeon, R. (2009). Which penguin is this? Attributing false beliefs about object identity at 18 months. Child Development, 80, 1172-1196.

Scott, R. M., \& Baillargeon, R (2013). Do infants really expect others to act efficiently? A critical test of the rationality principle. Psychological Science, 24, 466-474.

Scott, R. M., Baillargeon, R., Song, H. J., \& Leslie, A. M. (2010). Attributing false beliefs about non-obvious properties at 18 months. Cognitive Psychology, 61, 366-395.

Scott, R. M., Roby, E., \& Baillargeon, R. (in press). How sophisticated is infants' theory of mind? To appear in O. Houdé \& G. Borst (Eds.-in-chief), Cambridge handbook of cognitive 
development. Cambridge, England: Cambridge University Press.

Song, H. J., \& Baillargeon, R. (2008). Infants' reasoning about others' false perceptions. Developmental Psychology, 44, 1789-1795.

Southgate, V., \& Vernetti, A. (2014). Belief-based action prediction in preverbal infants. Cognition, 130, 1-10.

Spaepen, E., \& Spelke, E. (2007). Will any doll do? 12-month-olds' reasoning about goal objects. Cognitive Psychology, 54, 133-154.

Stavans, M., \& Baillargeon, R. (2018). 4.5-month-old infants individuate and track simple tools following functional demonstrations. Developmental Science, 21(1), e12500.

Stavans, M., Lin, Y., Wu, D., \& Baillargeon, R. (2019). Catastrophic individuation failures in infancy: A new model and predictions. Psychological Review, 126(2), 196-225.

Surian, L., Caldi, S., \& Sperber, D. (2007). Attribution of beliefs by 13-month-old infants. Psychological Science, 18, 580-586.

Ting, F., \& Baillargeon, R. (2020, July). 16-month-old infants perceive irrational individuals as having reduced moral rights. Paper presented at the virtual Biennial Meeting of the International Congress of Infant Studies.

Ting, F., Buyukozer Dawkins, M., Stavans, M., \& Baillargeon, R. (in press). Principles and concepts in early moral cognition. To appear in J. Decety (Ed.), The social brain: A developmental perspective. Cambridge, MA: MIT Press.

Ting, F., He, Z., \& Baillargeon, R. (2019). Toddlers and infants expect individuals to refrain from helping an ingroup victim's aggressor. Proceedings of the National Academy of Sciences, 116(13), 6025-6034.

Vouloumanos, A., Martin, A., \& Onishi, K. H. (2014). Do 6-month-olds understand that speech 
can communicate? Developmental Science, 17, 872-879.

Wang S., Baillargeon R, Brueckner L. (2004). Young infants' reasoning about hidden objects: Evidence from violation-of-expectation tasks with test trials only. Cognition, 93, 167-98.

Wang, S., \& Goldman, E. J. (2016). Infants actively construct and update their representations of physical events: Evidence from change detection by 12-month-olds. Child Development Research, article 3102481.

Wang, S., \& Onishi, K. H. (2017). Enhancing young infants' representations of physical events through improved retrieval (not encoding) of information. Journal of Cognition and Development, 18, 289-308.

Wang, S., Zhang, Y., \& Baillargeon, R. (2016). Young infants view physically possible support events as unexpected: New evidence for rule learning. Cognition, 157, 100-105.

Welder, A. N., \& Graham, S. A. (2001). The influence of shape similarity and shared labels on infants' inductive inferences about nonobvious object properties. Child Development, 72, $1653-1673$.

Wilcox, T. (1999). Object individuation: Infants' use of shape, size, pattern, and color. Cognition, $72,125-166$.

Woodward, A. L. (1998). Infants selectively encode the goal object of an actor's reach. Cognition, $69,1-34$

Woodward, A. L. (1999). Infants' ability to distinguish between purposeful and non-purposeful behaviors. Infant Behavior and Development, 22, 145-160. 
Figure 1

Familiarization Trials 1 and 2

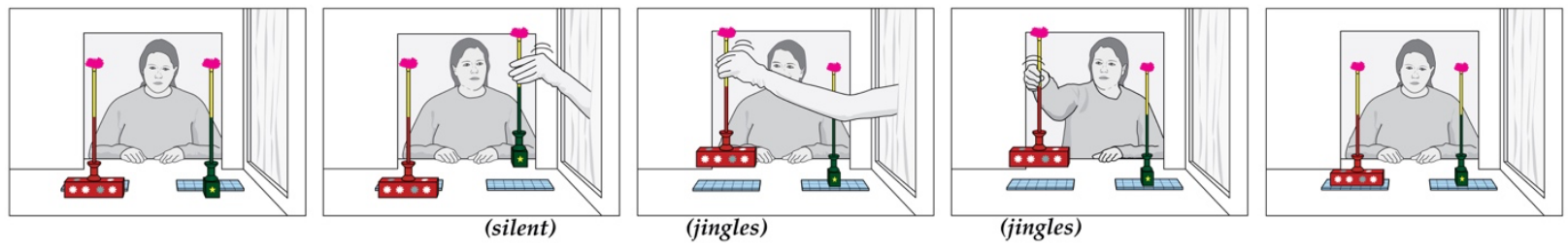

Familiarization Trials 3 and 4

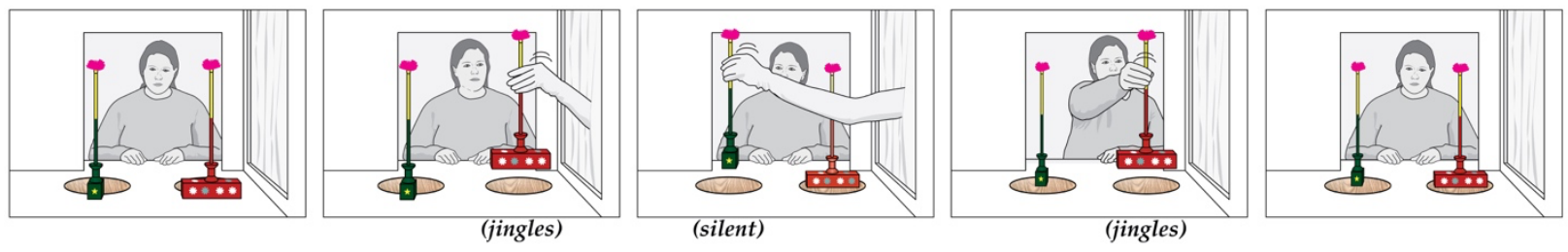

Figure 1. Familiarization Trials 1-4 for all four conditions. 


\section{Figure 2}

\section{Test Trial}

Inference Condition
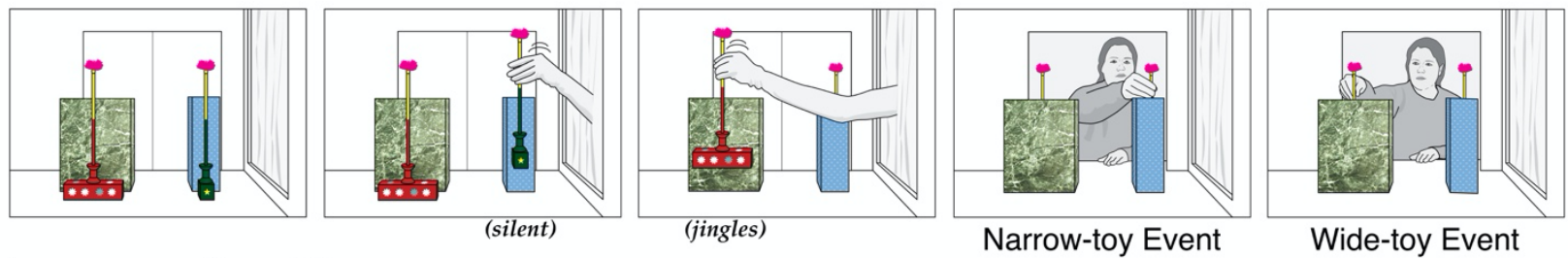

Ignorance Condition
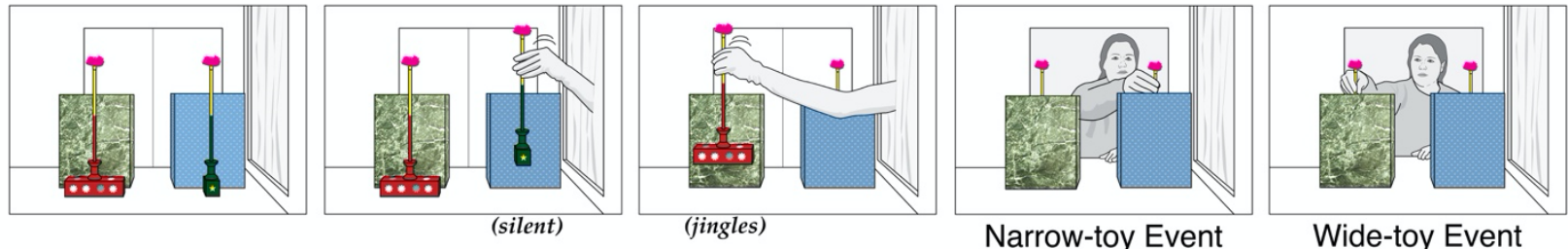

No-Preference Condition
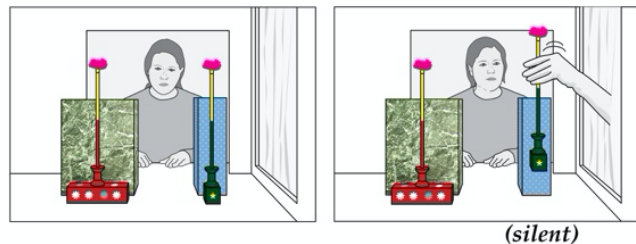

Perception Condition
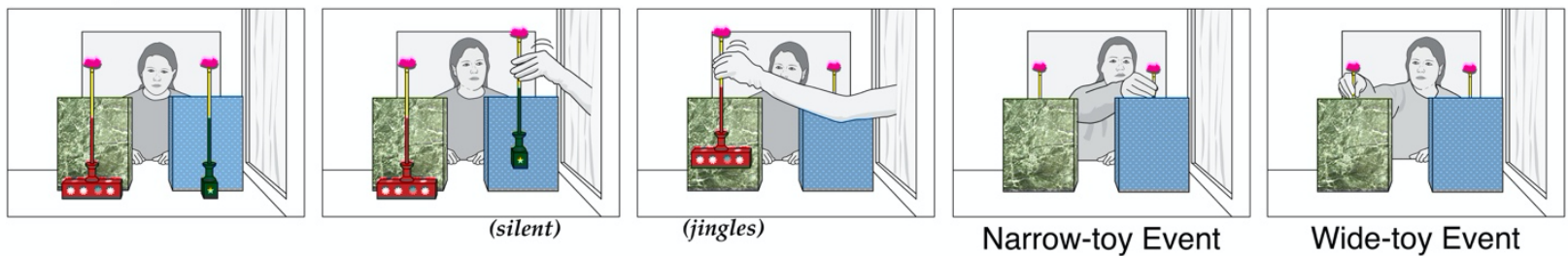

Figure 2. Test trial in each of the four conditions. Each infant saw either the wide-toy or the narrow-toy event. 
Figure 3

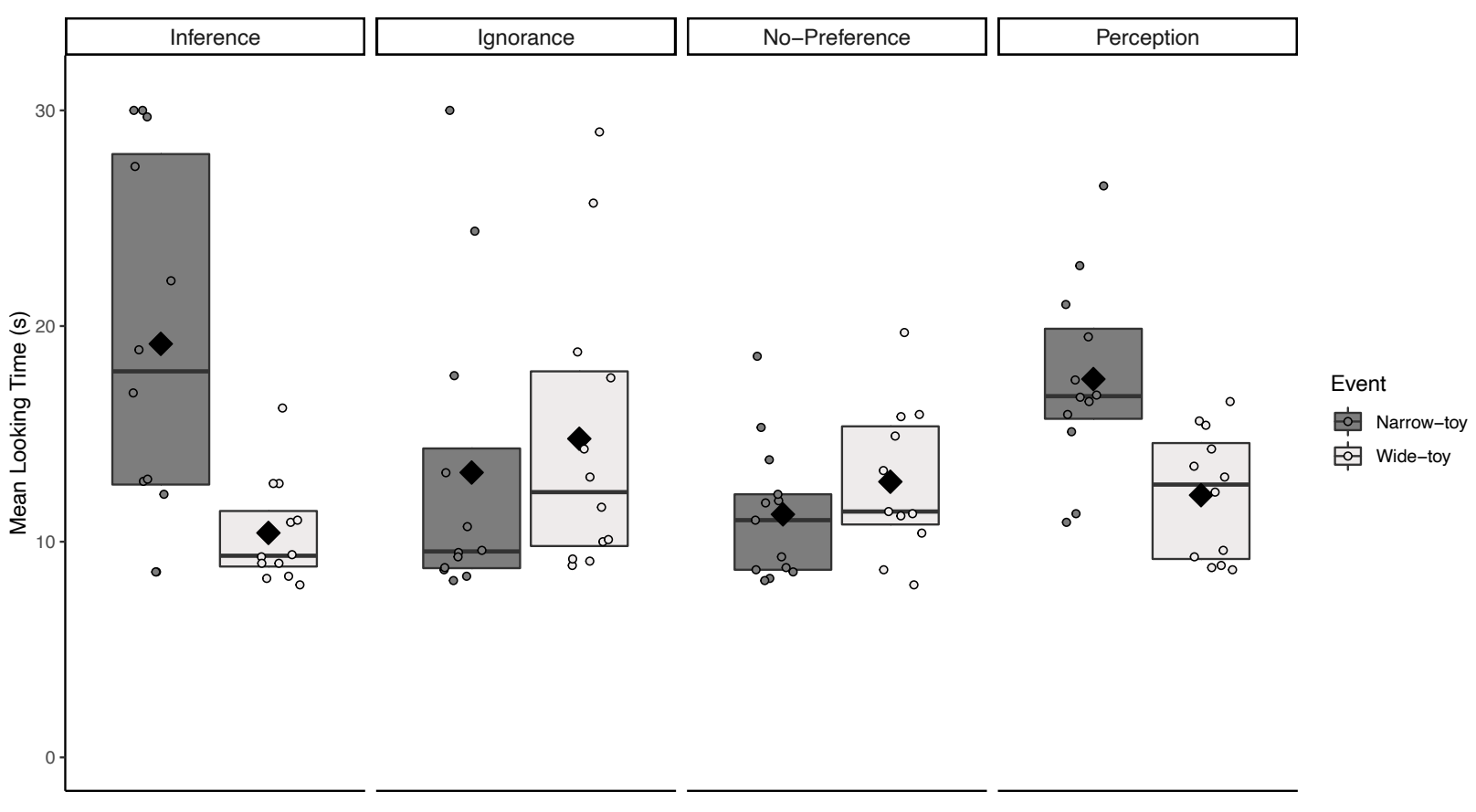

Figure 3. Looking times at the test events, by condition. Each dot represents a single infant; diamonds indicate means; boxes represent the interquartile ranges between the first and third quartiles; and the horizontal line inside each box indicates the median. 